Revue des patrimoines

\title{
Diversité patrimoniale des collections du haras du Pin : les enjeux de la conservation et de la valorisation des collections
}

\section{Muriel Meneux}

\section{OpenEdition}

Journals

Édition électronique

URL : http://journals.openedition.org/insitu/12397

DOI : 10.4000/insitu. 12397

ISSN : 1630-7305

Éditeur

Ministère de la culture

Référence électronique

Muriel Meneux, « Diversité patrimoniale des collections du haras du Pin : les enjeux de la conservation et de la valorisation des collections », In Situ [En ligne], 27 | 2015, mis en ligne le 23 novembre 2015, consulté le 21 avril 2019. URL : http://journals.openedition.org/insitu/12397 ; DOI : 10.4000/ insitu. 12397

Ce document a été généré automatiquement le 21 avril 2019.

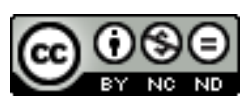

In Situ Revues des patrimoines est mis à disposition selon les termes de la licence Creative Commons Attribution - Pas d'Utilisation Commerciale - Pas de Modification 4.0 International. 


\title{
Diversité patrimoniale des collections du haras du Pin : les enjeux de la conservation et de la valorisation des collections
}

\author{
Muriel Meneux
}

\section{Les collections du haras du Pin}

1 Construit entre 1715 et 1730 par Pierre Le Mousseux selon les plans de Robert de Cotte, premier architecte du roi, architecte des Grandes Écuries de Versailles, sur un domaine de plus de 1000 hectares, le haras du Pin (fig. 1) a toujours été le fleuron du patrimoine des Haras nationaux. L'administration des Haras, créée en 1665 par Colbert, avait pour objectifs initiaux la production de races de chevaux, notamment pour la remonte (guerre, transport...) et l'amélioration génétique des races équines. 
Figure 1

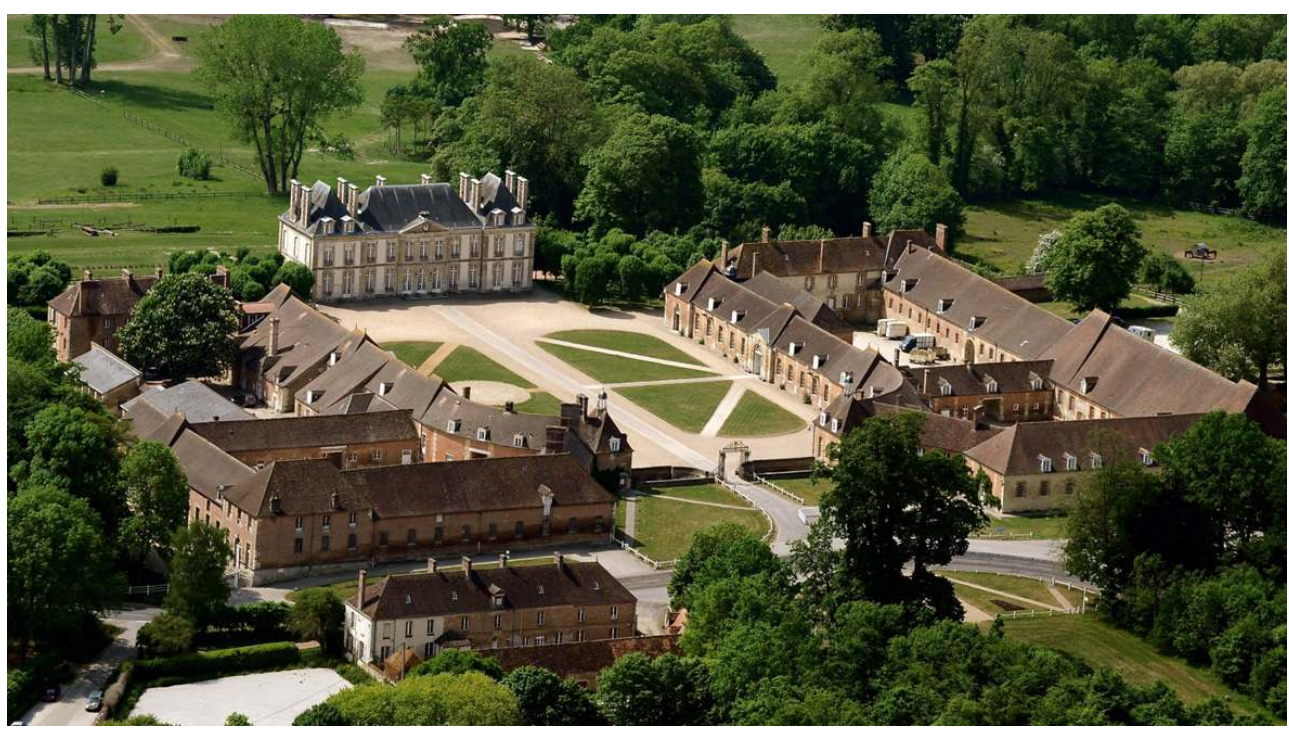

Vue aérienne du haras du Pin.

Phot. Rubio, Jean-Éric. ( Jean-Éric Rubio.

2 Selon la définition du Robert historique, le terme même de haras est apparu au XIII ${ }^{\mathrm{e}}$ siècle sous la forme " haraz », issu peut-être de l'ancien scandinave hârr, "qui a le poil gris ", d'après la couleur la plus fréquente de la robe des chevaux, ou de l'italien dialectal ara, " abri pour les chevaux".

3 Très tôt, la langue a attribué un mot spécifique à l'élevage du cheval et ce, dès le Moyen Âge.

4 Suite à la création de l'administration des Haras royaux en 1665, le premier haras royal créé fut celui de Saint-Léger-en-Yvelines mais les conditions d'élevage s'avérèrent peu favorables. Qui plus est, le domaine de ce haras royal appartenant au duc de Chevreuse, le roi devait lui verser une somme de 3000 livres de dédommagement par an.

5 En 1714 fut confiée à François Gédéon de Garsault, capitaine du haras royal, la mission de chercher un nouveau lieu. La Normandie étant reconnue comme terre d'élevage du cheval depuis le Moyen Âge, Garsault choisit la terre du «Buisson d'Hiesmes», propriété du marquis Béchameil de Nointel, maître des requêtes et alors intendant de Riom. En 1715, le transfert officiel du haras royal eut lieu sur cette nouvelle terre normande, avec la création du haras du Pin. En 1717, 200 chevaux arrivèrent dans les nouvelles écuries.

6 Cette mission nécessitait de trouver une terre propice à l'élevage, une terre de tradition, de construire une architecture apte à héberger et à faire travailler des chevaux mais aussi les hommes qui s'en occupent, de mettre en place des métiers essentiels à la vie du cheval et par la suite, de faire évoluer ces métiers par rapport aux techniques d'élevage.

7 Toutes les collections sont donc nées à partir des outils de production des chevaux, certaines étant davantage liées à la fonction de représentation du haras du Pin.

8 La période de la Révolution entraîna une pénurie de chevaux. Le haras du Pin subsista malgré la demande de fermeture formulée par l'assemblée départementale dès 1789.

9 Grâce au développement des courses, le haras, devenu impérial en 1806, connut des années de développement en participant activement à la détermination de deux races, le 
pur-sang anglais et le trotteur français. Cet essor se poursuivit sous le Second Empire, marqué par la visite de Napoléon III en 1863 venu assister aux courses sur l'hippodrome de la bergerie.

10 Cette phase d'expansion au XIX ${ }^{e}$ siècle s'accompagna de nouvelles constructions: les bâtiments de l'hippodrome, la succursale des Charmettes et celle de Borculo, la cité Pontavice pour le personnel du haras, la mairie-école... (fig. 2)

Figure 2

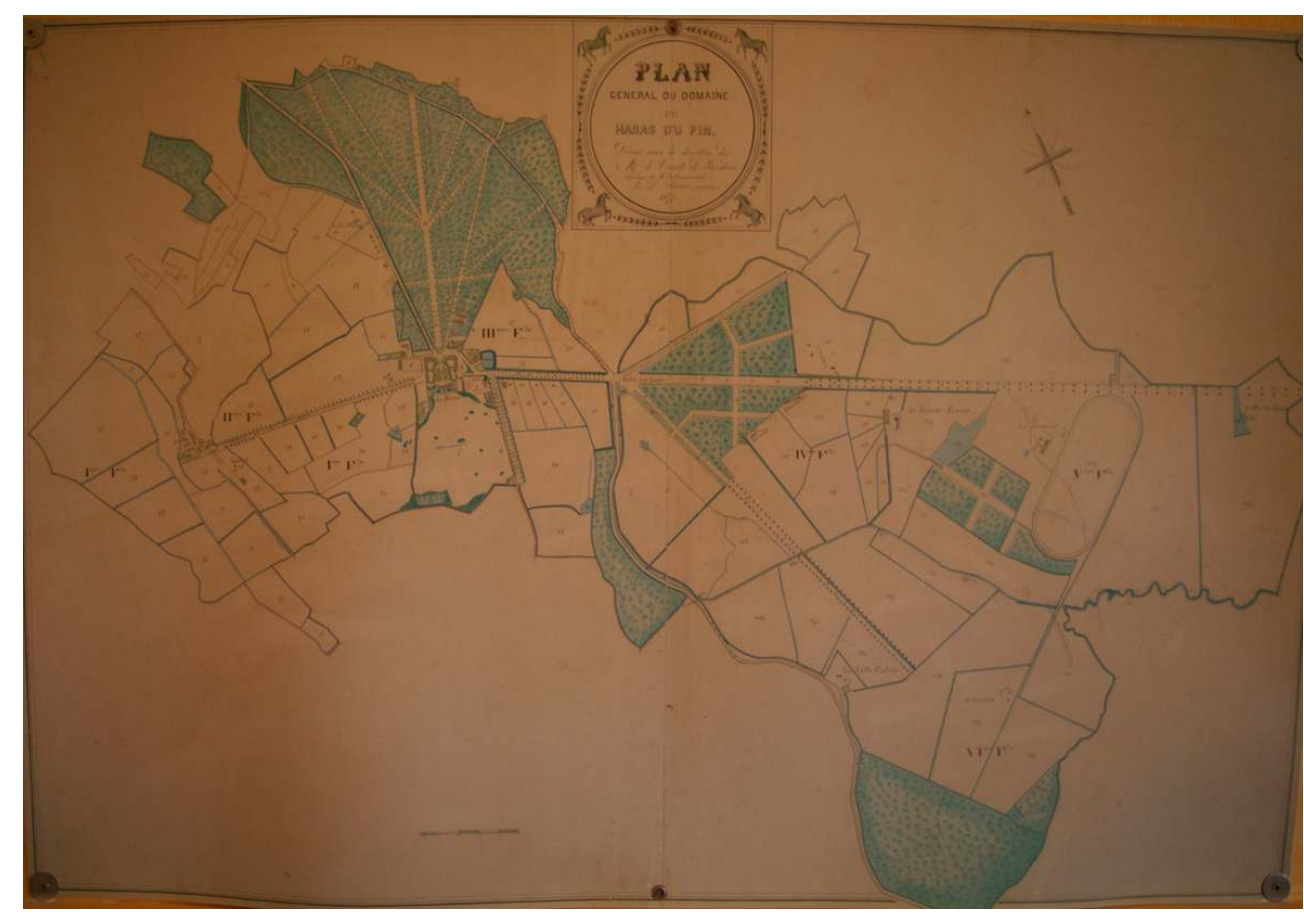

Plan général du domaine du haras du Pin. Dressé sous la direction de M. le comte de Pardieu, directeur de l'établissement, par L. Bezier, instituteur, 1877.

(c) Haras du Pin Tourisme.

11 En 1844, après le cheval, la vache fut introduite sur le domaine du Pin. Plus proche de nous, dans les années 1990, la jumenterie et les écuries du bois ont été transformées en centre de formation tandis qu'en 2006, le haras ouvrait un centre d'interprétation du patrimoine et un espace d'accueil pour les visiteurs.

12 Après des changements et des réformes enclenchées dès le début des années 1990, les Haras nationaux forment un établissement public, l'Institut français du Cheval et de l'Équitation (IFCE), auquel s'est adjointe depuis 2010 l'École nationale d'équitation de Saumur.

13 Les missions régaliennes d'élevage du cheval se sont peu à peu réduites dans les haras nationaux. Les nouvelles activités liées aux compétitions sportives, à l'événementiel en général et au tourisme ont supplanté les missions d'élevage dévolues aujourd'hui à France-Haras. Le haras du Pin demeure un emblème dans le milieu du cheval mais peut-il rester uniquement une figure symbolique alors qu'il se situe dans la première région d'élevage du cheval en France?

Nous essaierons de vous démontrer l'identité du lieu marquée par la diversité des collections patrimoniales du haras du Pin pour donner une vision plus complète de sa 
richesse. Pour donner une vision plus complète de sa richesse nous ferons parallèlement un état des lieux de la conservation et une analyse des enjeux de la valorisation, sur un plan à la fois culturel et économique, pour les Jeux équestres mondiaux de 2014. Avant d'aborder les collections proprement dites, il est essentiel de resituer le haras dans le contexte de sa mission, l'élevage du cheval, qui a bénéficié d'un environnement paysager exceptionnel.

\section{Le cheval, destinée du haras}

Cette politique en faveur de l'élevage du cheval, voulue par le roi de France, fut déterminante pour la destinée du haras du Pin.

Figure 3

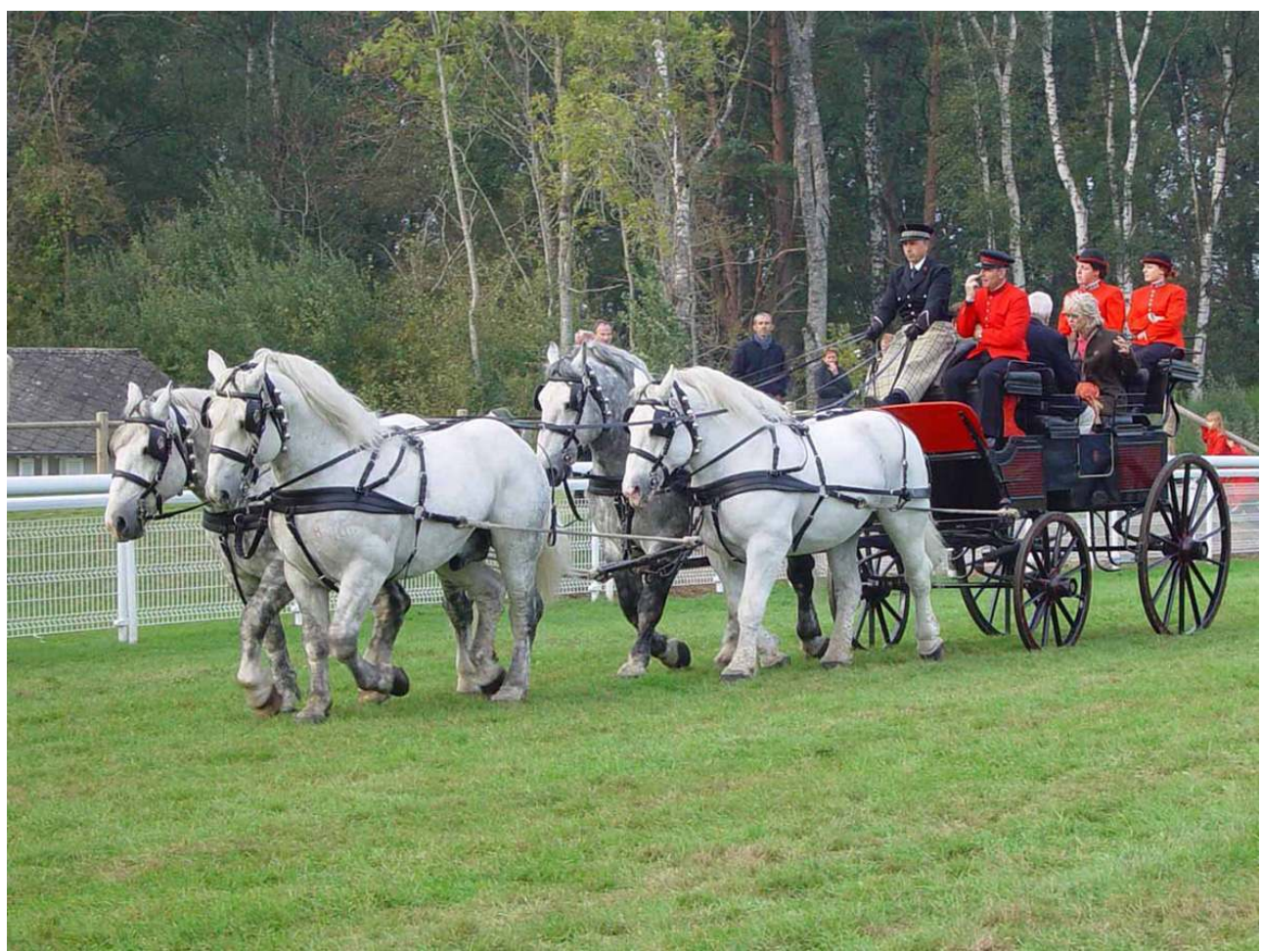

Attelage de percherons sur l'hippodrome du Pin.

Coll. part. 
Figure 4

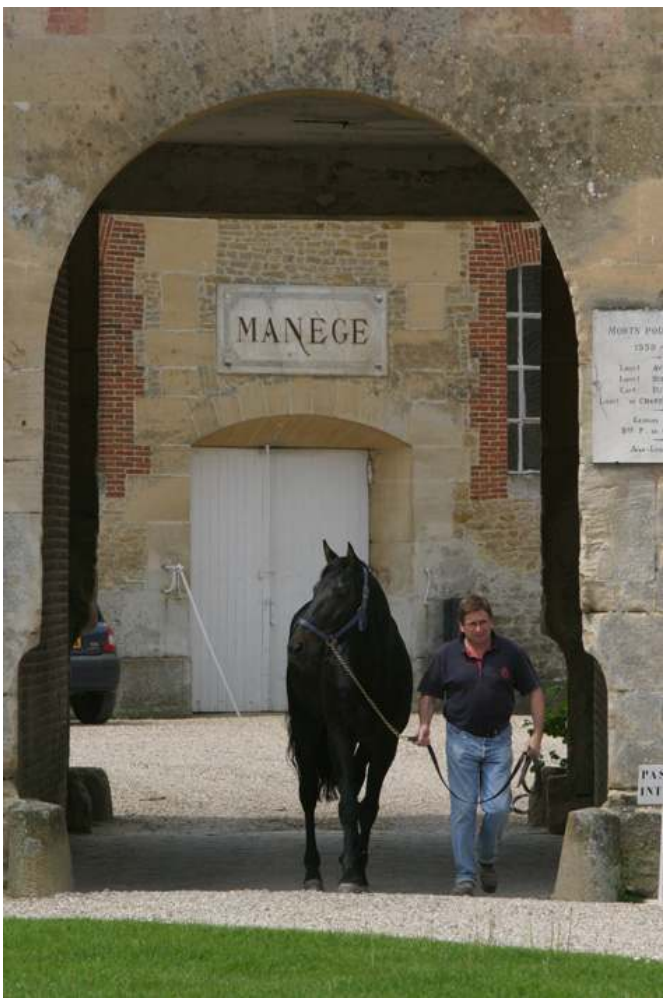

Étalon trotteur français revenant de la récolte. (c) Agence Dollar. 


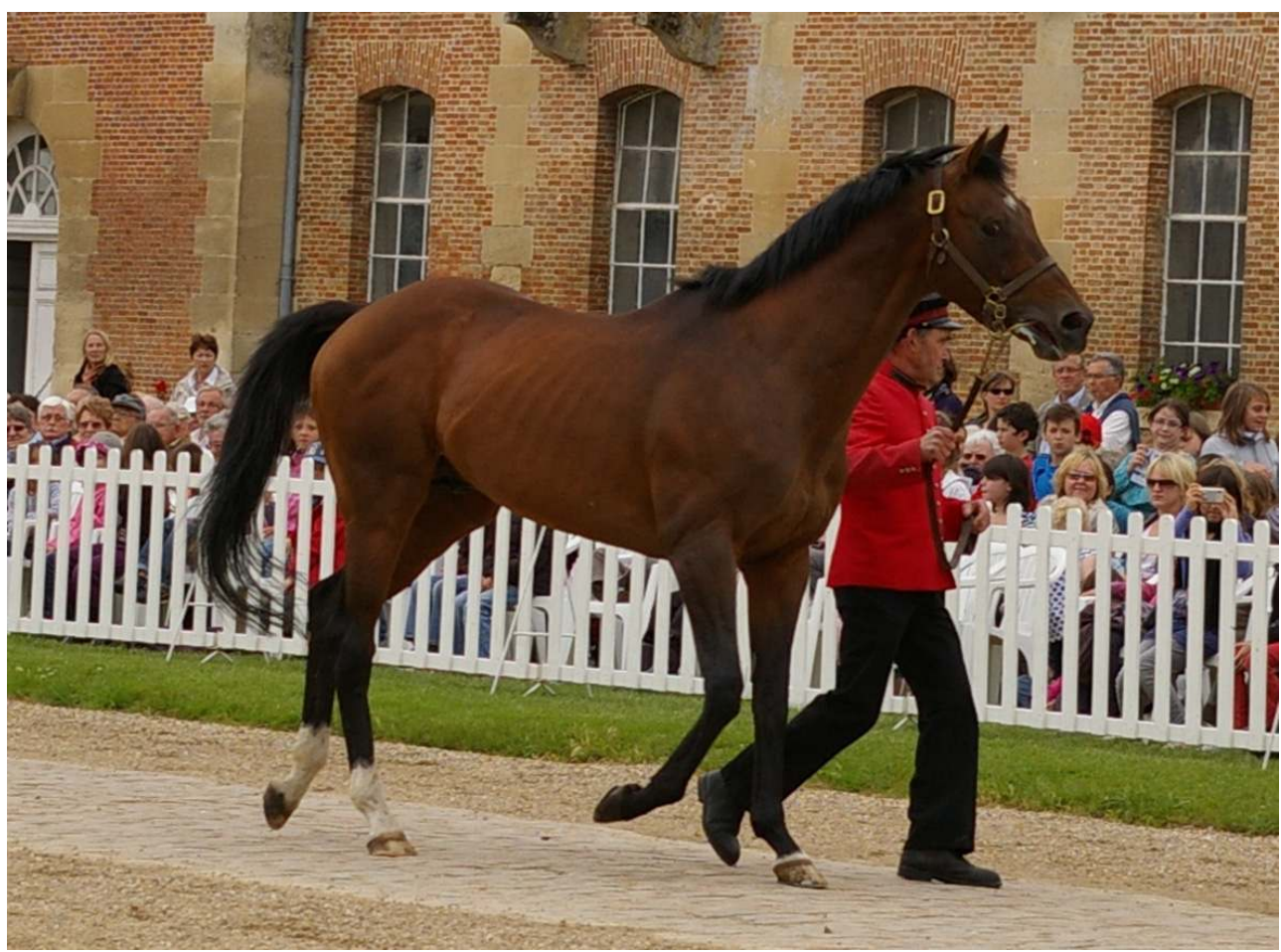

Étalon pur-sang anglais.

(C) Haras du Pin Tourisme.

16 Le haras du Pin est le berceau de trois races, le percheron (fig. 3), le trotteur français (fig. 4) et le pur-sang anglais (fig. 5). Ces deux races de chevaux de course expliquent la notoriété acquise par le haras grâce à des étalons devenus des chefs de race, un véritable patrimoine vivant génétique. Nino des Buissonnets, qui a gagné le concours de saut d'obstacles aux Jeux olympiques de Londres, est un arrière-arrière-petit-fils de Furioso, grand chef de race au haras du Pin. On note aujourd'hui l'absence d'étalons dans les écuries. Par contre, il reste des chevaux pour les formations et les présentations et des races étrangères y sont maintenant hébergées comme le barbe (fig. 6), le nonius ou encore le lusitanien. 
Figure 6

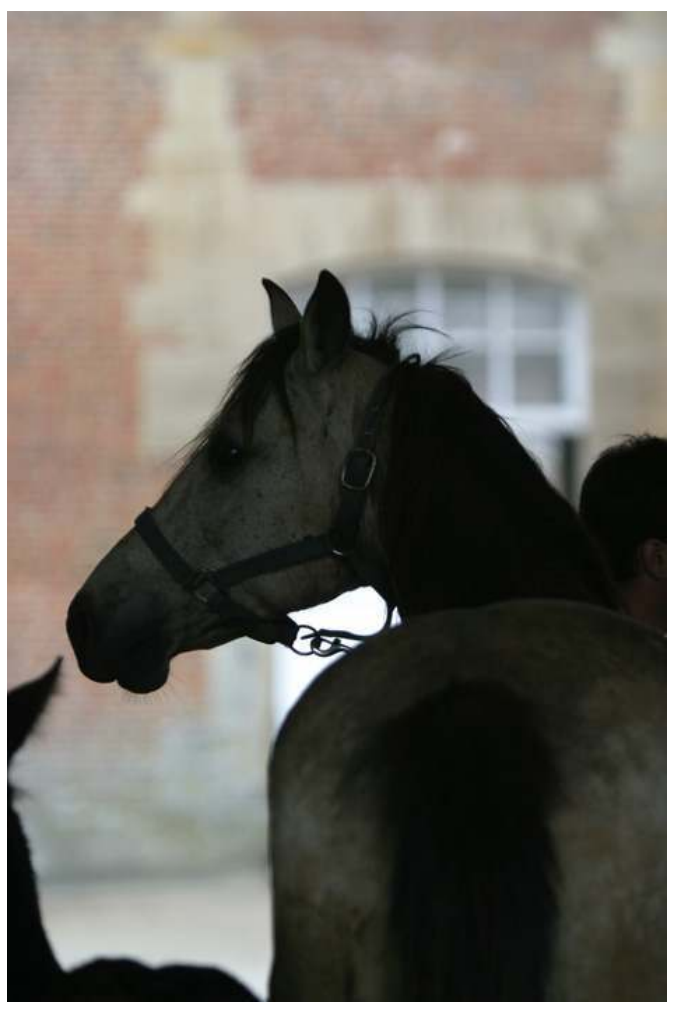

Étalon barbe.

(c) Agence Dollar.

Pourtant, nous devons préserver un patrimoine animal, notamment des races de chevaux de trait et des ânes. Quand le sénateur Larcher rédigeait en 1994 un premier rapport sur «Patrimoine animal, patrimoine rural. Contribution à la vie du territoire», cela apparaissait comme une alerte puisque celui-ci évoquait «la déperdition d'éléments patrimoniaux ", relevant la situation préoccupante pour les races équines.

18 Faut-il conserver ce patrimoine vivant dans ce lieu en acceptant d'en perdre la partie génétique ou le faire évoluer avec une autre structure d'élevage?

D'autre part, il s'agit de renforcer le patrimoine pédagogique en terme de formation de l'attelage à l'équitation, en adéquation avec les emplois offerts par la filière cheval, y compris sur le plan international. Le haras du Pin doit rester au service des éleveurs et de l'ensemble des professionnels de la filière équine.

\section{Le patrimoine paysager, la genèse du haras du Pin}

Sans cette terre d'élevage, la construction du haras du Pin n'aurait jamais été envisagée. C'est bien ce territoire de pâtures, de forêts et de milieux humides qui a favorisé son implantation.

21 Le premier plan du domaine fut dressé en 1736, en lien avec une fonction précise, la production de chevaux pour le roi (fig. 7). En 2012, c'est cette même limite qui crée l'identité domaniale (étude de la DIREN, 1997). Deux axes majeurs nous font penser au decumanus et au cardo de la ville romaine, l'avenue Louis XIV et l'allée de l'hippodrome 
mais ce n'est pas le forum l'espace central mais le haras, avec sa cour d'honneur et ses bâtiments. C'est lui qui est au cœur de la vie du domaine qui a été bâti à partir des besoins d'élevage du cheval.

Figure 7

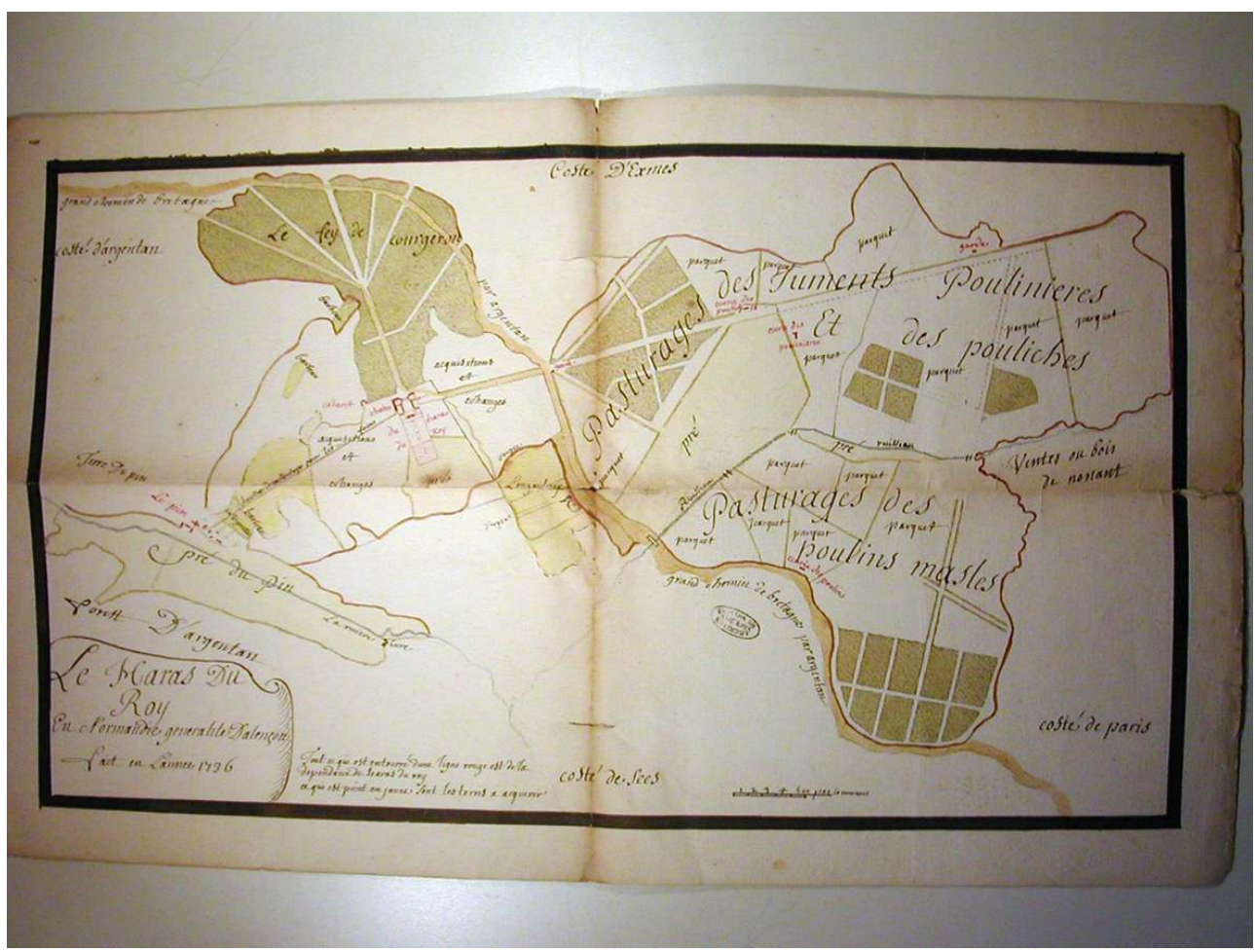

Plan du haras du roy en Normandie. Généralité d'Alençon, 1736. Archives nationales, Paris, Q1-186.

(c) Haras du Pin Tourisme.

En 1997, la direction régionale de l'Environnement, de l'Aménagement et du Logement (ex-DIREN) a rédigé une étude paysagère sur le haras du Pin, un état des lieux repris par l'École nationale supérieure du paysage de Versailles, qui a privilégié en 2012 une étude sur la valorisation du centre historique du domaine du Pin et du parcours de cross pour les Jeux équestres mondiaux de 2014, à la demande de l'Institut français du Cheval et de l'Équitation.

\section{Le patrimoine architectural du haras du Pin}

Véritable innovation de l'architecture équestre, le haras du Pin demeure une construction royale inédite mais avant tout fonctionnelle, pour héberger les chevaux, au décor sobre et très empreinte de l'architecture de l'école de Versailles. Si la légende a attribué pendant des années la construction du haras à Jules Hardouin-Mansart et les jardins à Le Nôtre, des recherches effectuées par Jean-Louis Libourel ont fait connaître le nom de deux architectes : Pierre Le Mousseux et Robert de Cotte. Dessiné selon un plan en fer à cheval, l'ensemble des bâtiments s'ordonne autour de la cour d'honneur. Des agrandissements ont été réalisés au XIX ${ }^{\mathrm{e}}$ siècle ${ }^{1}$.

La priorité des Haras nationaux fut dès l'origine de renouveler le patrimoine génétique équin, pas de s'intéresser à son patrimoine architectural. Même si des urgences ont été 
définies, nous sommes loin d'une politique d'entretien et de restauration patrimoniales des bâtiments et autres au haras du Pin, à moyen terme et à long terme.

\section{Les collections mobilières, constitution et caractérisation}

Le haras du Pin a réuni des objets et des documents nécessaires à sa mission première, l'élevage du cheval. Ces objets et documents, production endogène du haras, étaient utilitaires; ils ont constitué le fonds du haras du Pin, des collections au sens patrimonial du terme, au vu de leur intérêt historique, équestre, ethnographique, esthétique et muséographique.

\section{Les collections, productions de l'outil de travail}

\section{Le patrimoine écrit et iconographique}

Des fiches d'étalons aux carnets de saillies, aux registres tenus mentionnant les stations de monte avec le nom des étalons, aux registres avec le lieu et la ration d'alimentation des étalons sur le domaine du Pin ont formé l'outil de travail administratif du haras (fig. 8, fig. 9).

Figure 8

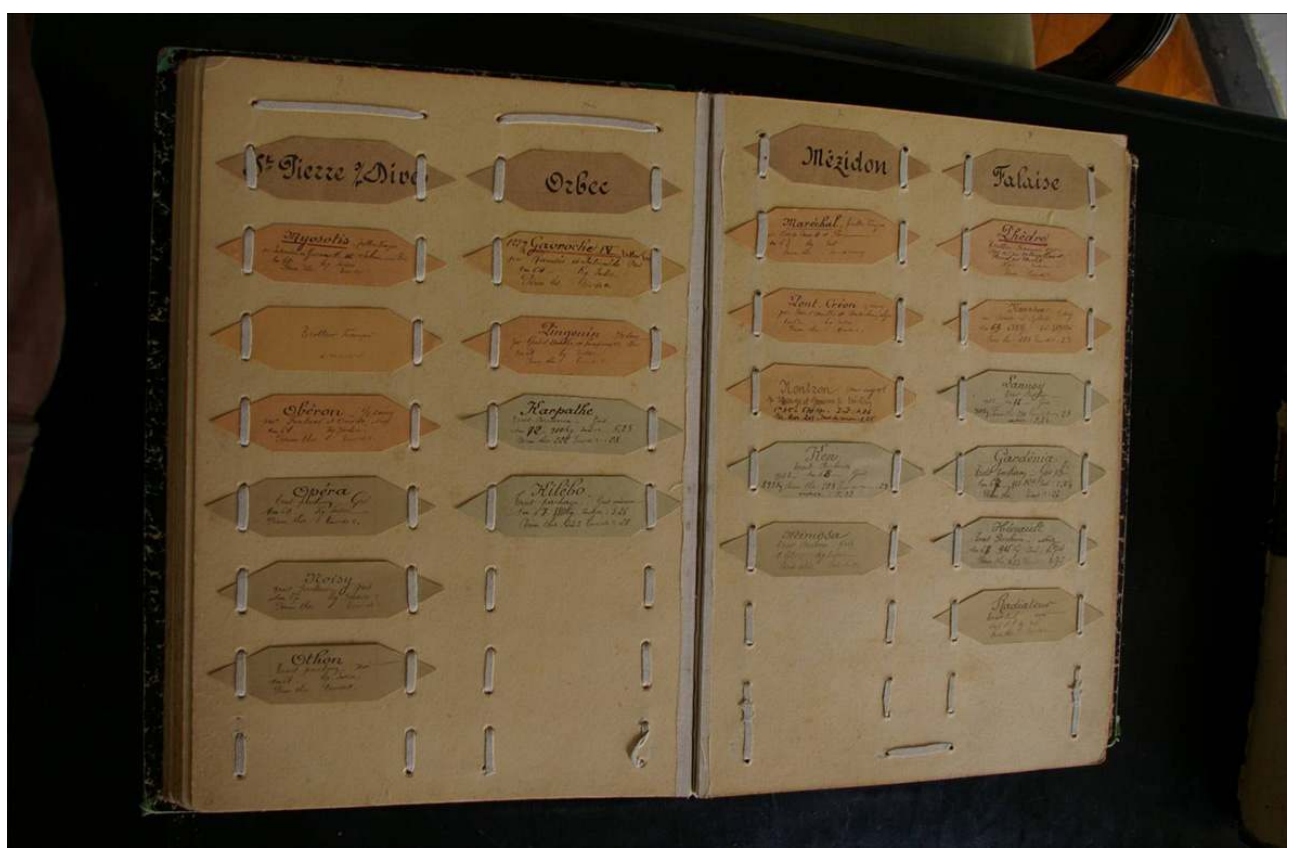

Registre des étalons pour la monte.

(c) Haras du Pin Tourisme. 
Figure 9

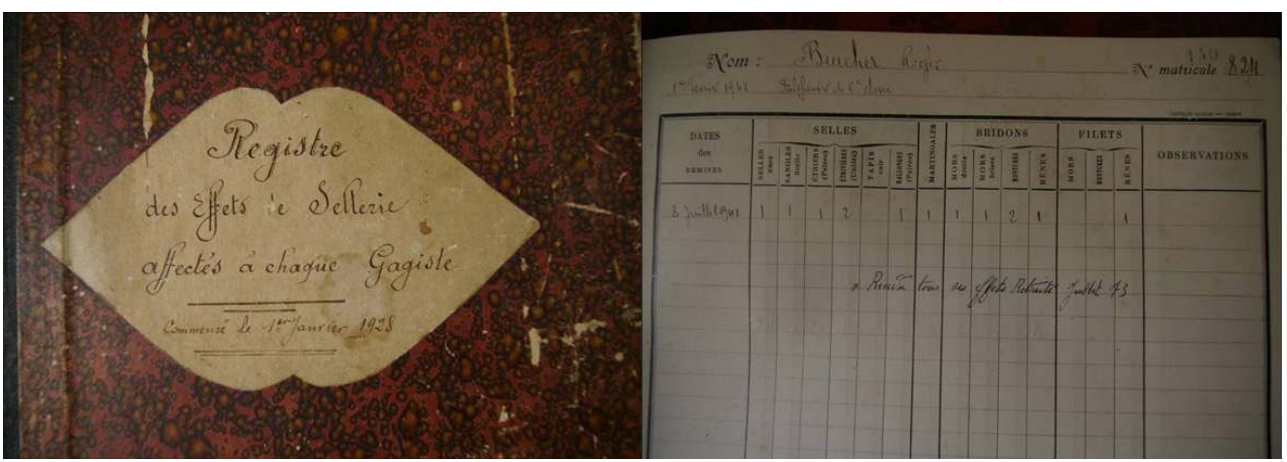

(A) REgISTRE des EFFETS de SELLERIE AFFECTÉS À CHAQUE gAgISTE, COMMENCÉ LE 1ER JANVIER 1928 (COUVERTURE). (B) EXTRAIT DU REgISTRE DES EFFETS DE SELLERIE AFFECTÉS À CHAQUE gAgISTE. ÉTABLI LE 1ER FÉVRIER 1942 AU PALEFRENIER de 6E CLASSE ROger BeUCHER, $\mathbf{N}^{\circ}$ MATRICULE : 824.

(C) HARAS DU PIN TOURISME.

La naissance de la photographie en 1839 a permis une nouvelle promotion de l'étalon, apparue à la fin du XIX $X^{\mathrm{e}}$ siècle avec la carte postale. Certes, quelques peintures ont mis en valeur les étalons, comme celles de Paul Le More (1863-1914) (fig. 10).

Figure 10

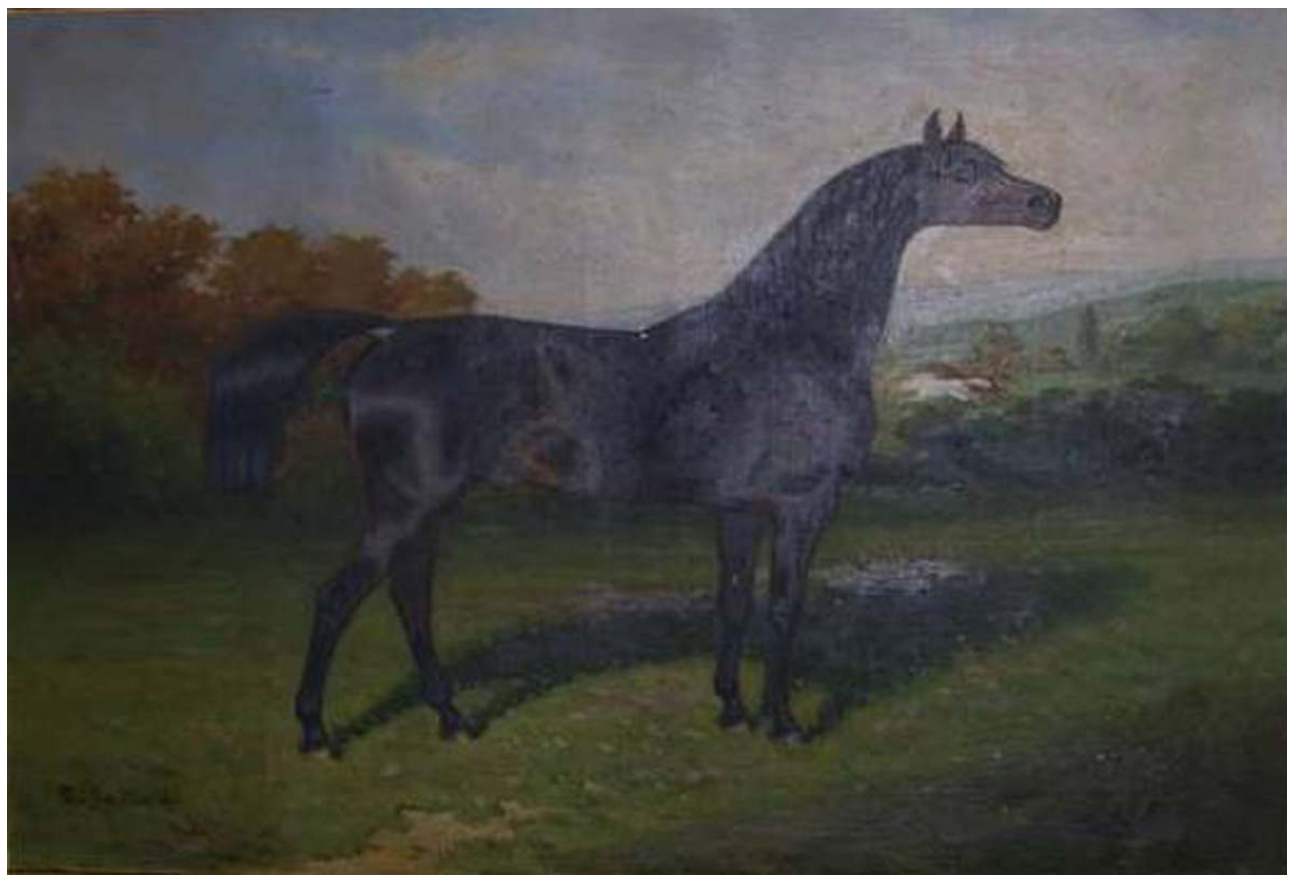

"Moustique, par Sting et Ester », Paul Le More (1863-1914), non daté, huile sur toile, situé dans le Parcours découverte de l'écurie $n^{\circ} 1$. Propriété des Haras nationaux-Haras du Pin.

(c) Haras du Pin Tourisme.

Si on peut observer une permanence de l'iconographie de l'étalon au haras du Pin depuis la fin du $\mathrm{XIX}^{\mathrm{e}}$ siècle, le sujet photographié de profil pour rehausser ses aplombs, sa morphologie, les oreilles dressées, on constate plutôt aujourd'hui l'absence des étalons au haras. Des cartes postales aux catalogues d'étalons, le métier de photographe équestre est 
une spécialité à part entière ; sans une bonne connaissance des chevaux, il paraît fort peu probable que l'on obtienne des photos aussi techniques.

L'ensemble de la correspondance administrative depuis le XVIII ${ }^{\mathrm{e}}$ siècle constitue la base des archives, reflétant les activités du haras, de ses missions, révélant parfois des pratiques et des savoir-faire aujourd'hui disparus.

Ce n'est d'ailleurs pas un hasard si le haras du Pin a été le premier des haras nationaux à faire part de son intention, dès 2005, d'inventorier son patrimoine même si le versement des archives n'allait pas de soi. C'est, sans doute, le fait de produire son propre fonds pour ses propres objectifs que les Haras nationaux protégeaient; la phase de versement des archives et de récolement des objets avec le statut de collections qu'ils acquéraient, signifiait, en partie, que leur valeur patrimoniale était établie : bien commun de tous, élément d'héritage et source historique. Aujourd'hui, les archives du haras du Pin ont été versées en majeure partie aux archives départementales de l'Orne.

31 Le haras, et surtout l'École nationale professionnelle des haras, installée au Pin dès 1841, a toujours disposé d'une bibliothèque pour ses élèves. C'est à présent une médiathèque, située dans les locaux actuels du centre de formation, ouverte à tous et en particulier aux stagiaires et aux agents qui y suivent une formation professionnelle.

\section{Le patrimoine équestre, base du travail des étalons}

\section{Objets et savoir-faire, patrimoine matériel et immatériel indissociable}

Au-delà de la mission d'élevage du cheval, il s'agissait d'entretenir et d'exercer les étalons.

Des gestes se sont perdus comme faire une tresse de paille tout le long d'écuries en stalles, par disparition des savoir-faire, mais aussi parce que depuis 1991, tous les chevaux sont dans des boxes et non plus dans des stalles.

\section{Les voitures hippomobiles}

Grâce au travail de Jean-Louis Libourel, c'est sans doute un des domaines du patrimoine du haras du Pin le plus connu, le mieux inventorié, et le mieux conservé avec, dernièrement la restauration de voitures comme le milord et le road-coach. Mais c'est aussi le meneur qui peut conduire ces voitures et être encore formé au centre de formation du Pin (fig. 11, fig. 12). 
Figure 11

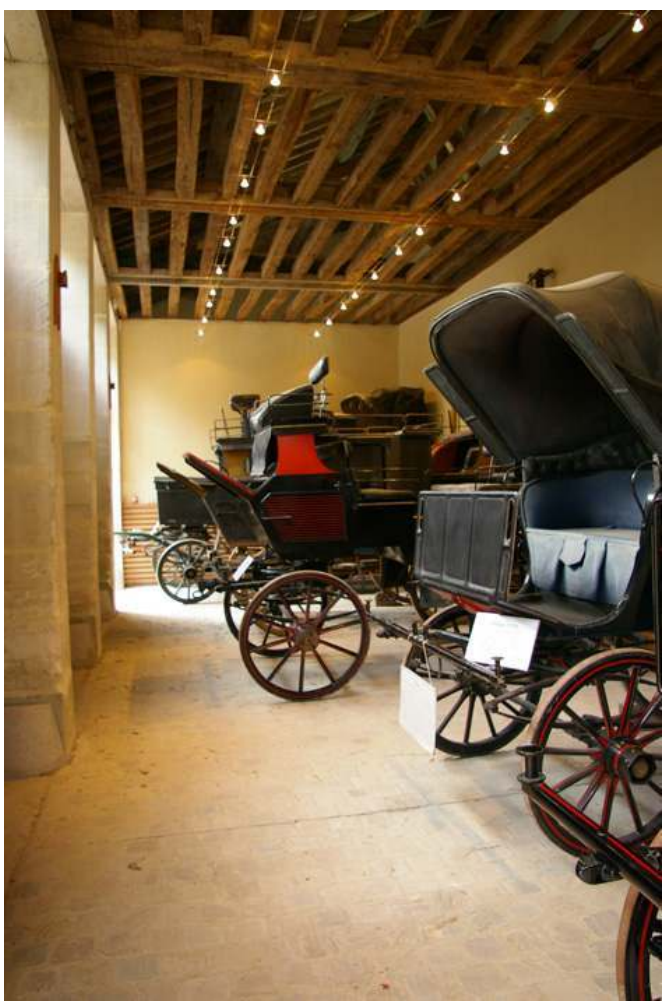

Remise à voitures hippomobiles restaurée en 2011-2012

(c) Haras du Pin Tourisme.

Figure 12

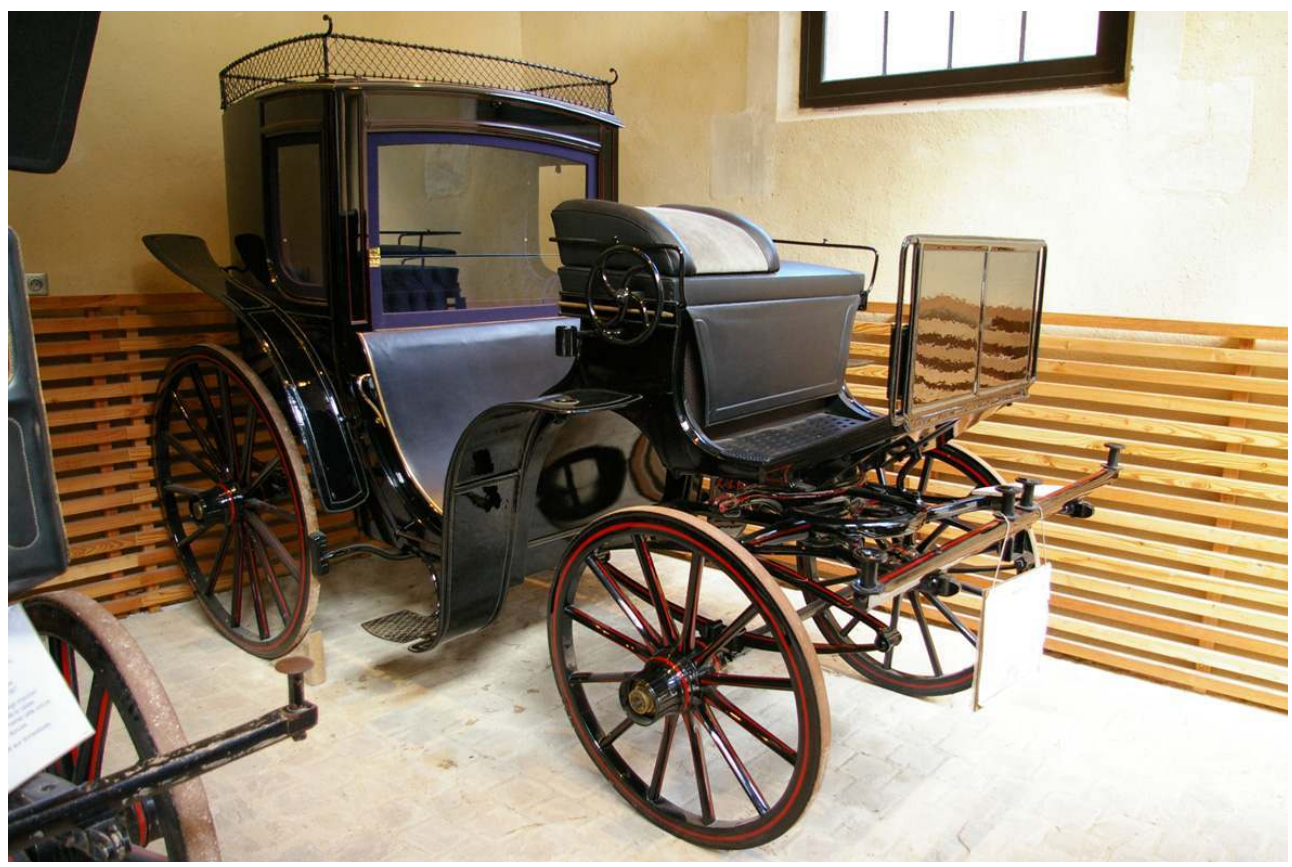

« Le Milord Fermé » restauré en 2010.

(c) Haras du Pin Tourisme. 


\section{Les selleries}

Le haras compte trois selleries: une sellerie d'honneur et deux selleries de travail, réunissant aussi bien les harnais pour l'attelage que les selles d'équitation, et le matériel pour les voitures hippomobiles (fig. 13, fig. 14). Ces selleries ont fait l'objet d'un premier inventaire très simple mais elles nécessitent aujourd'hui une sélection de ce qui doit être conservé et valorisé et devraient surtout bénéficier d'un inventaire patrimonial en bonne et due forme.

Figure 13

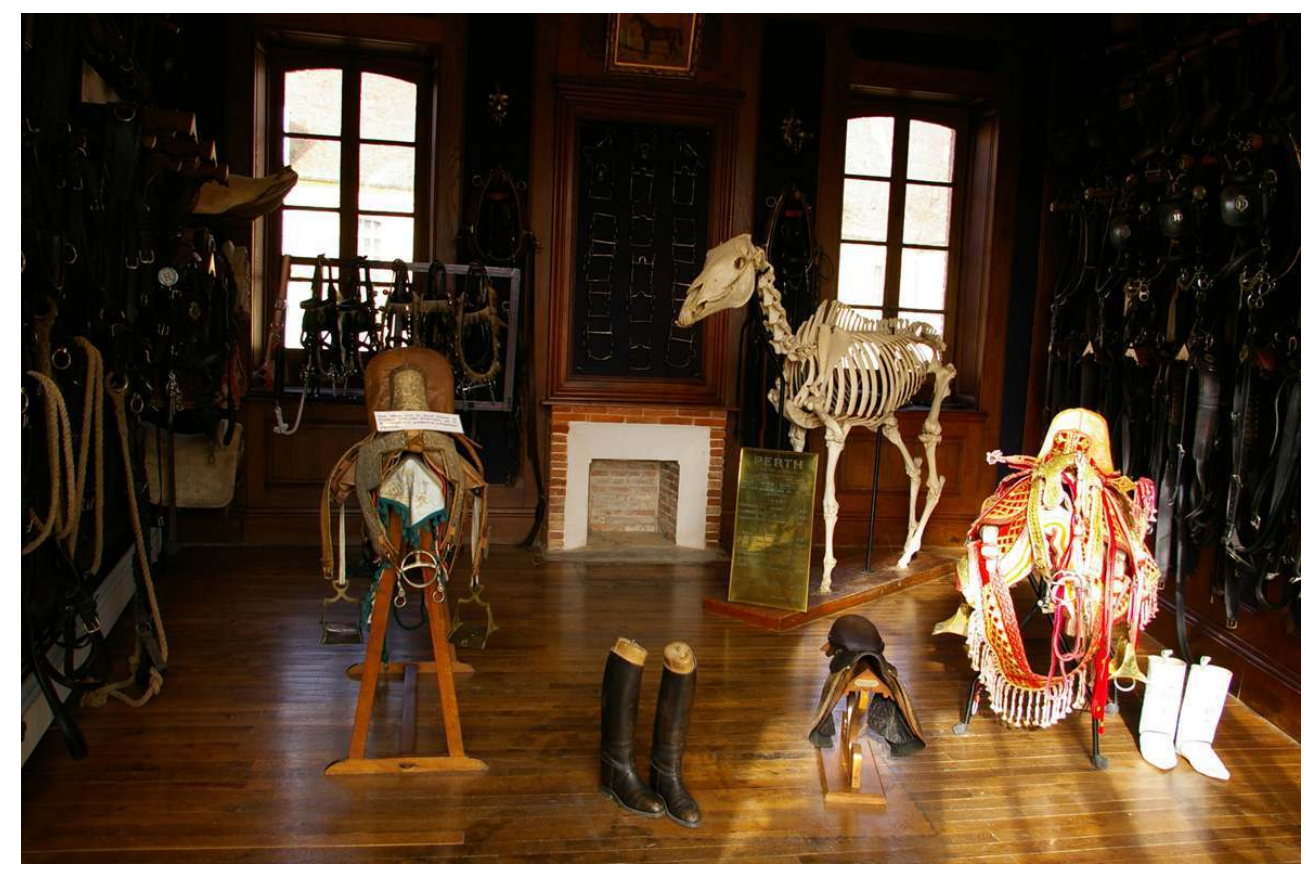

Sellerie d'honneur du haras du Pin.

(c) Haras du Pin Tourisme. 
Figure 14

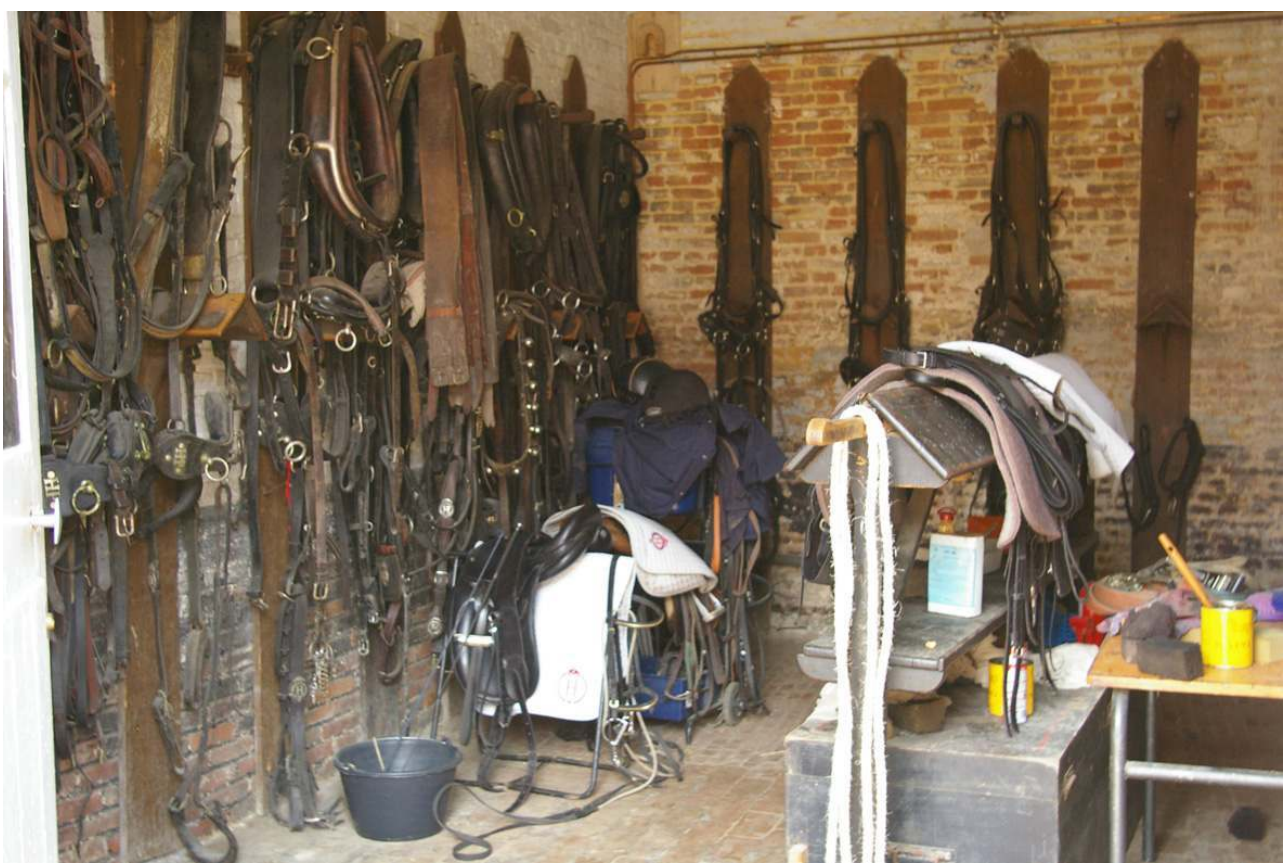

Sellerie de travail du haras du Pin

(c) Haras du Pin Tourisme.

Outre l'atelier de sellerie du haras (fig. 15), le centre de formation dispose d'un atelier de formation.

Figure 15

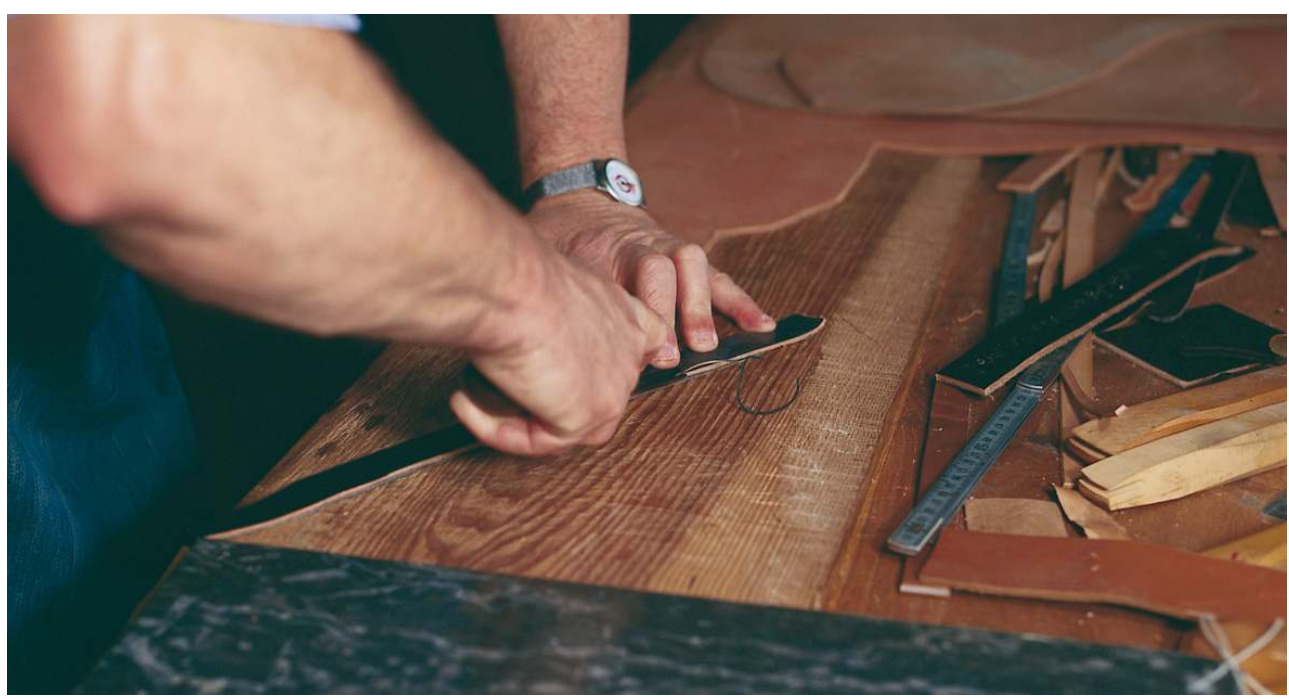

Sellier du haras du Pin dans son atelier.

Coll. part.

\section{La maréchalerie}

37 Nous retrouvons le même principe que pour la sellerie : une maréchalerie au haras et un atelier de formation de maréchalerie au centre de formation (fig. 16). Maréchal et sellier 
disposent d'outils propres à chacun de leurs métiers, de gestes et de savoir-faire ancestraux.

Figure 16

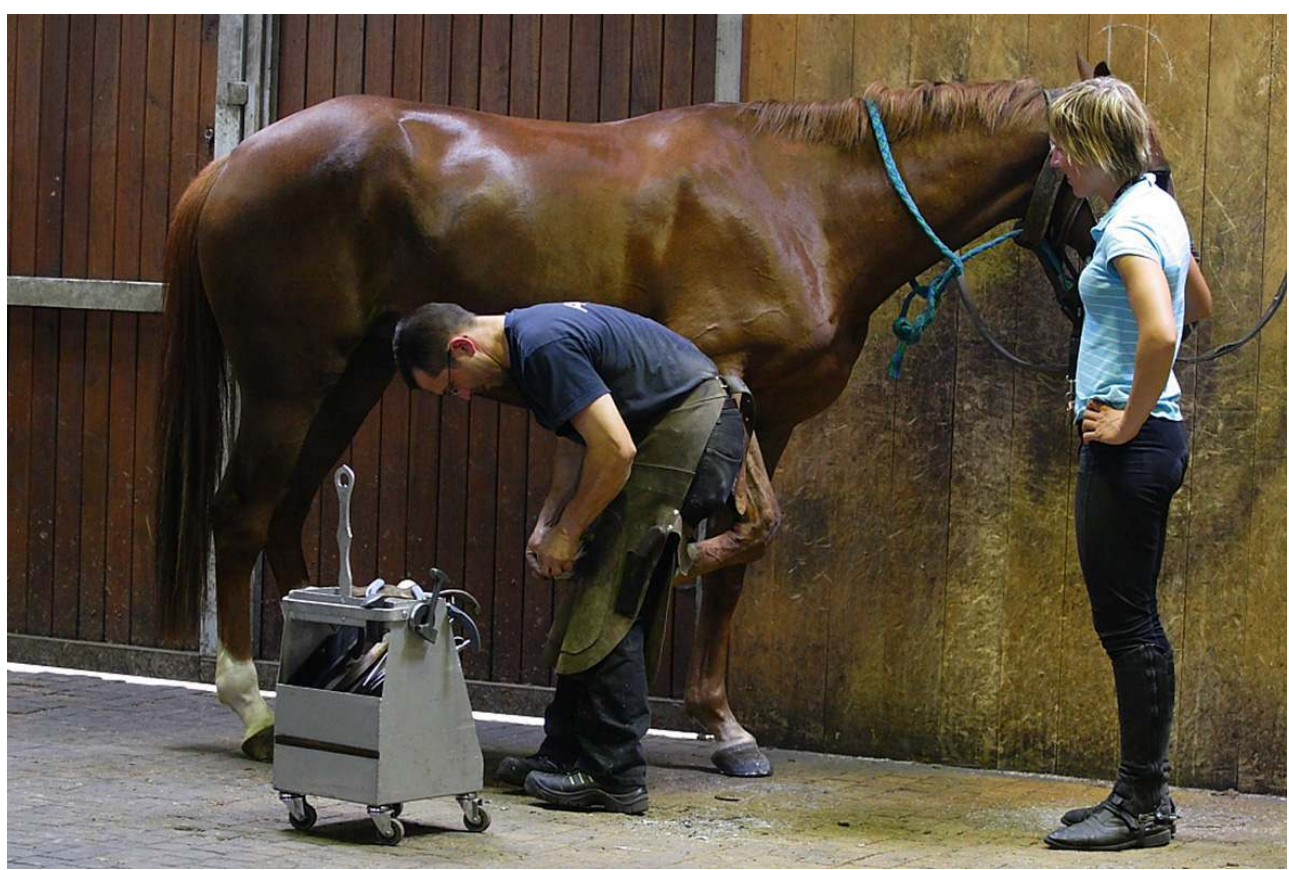

Maréchal-ferrant du haras du Pin.

Coll. part.

\section{L'émergence de nouveaux métiers}

Suite aux recherches scientifiques sur l'insémination artificielle et à son utilisation officielle pour le cheval dès 1985, de nouvelles compétences ont émergé : insémination, échographie mais aussi transfert d'embryons. Les Haras nationaux ont pu ainsi développer toute leur expertise en matière de formation sur ces métiers. Depuis une dizaine d'années, ce sont des activités professionnelles liées à l'intégration du cheval dans la société civile, mais aussi sa place dans le contexte de redécouverte de l'environnement, comme une spécialisation de cocher, qui sont apparues (fig. 17). 
Figure 17

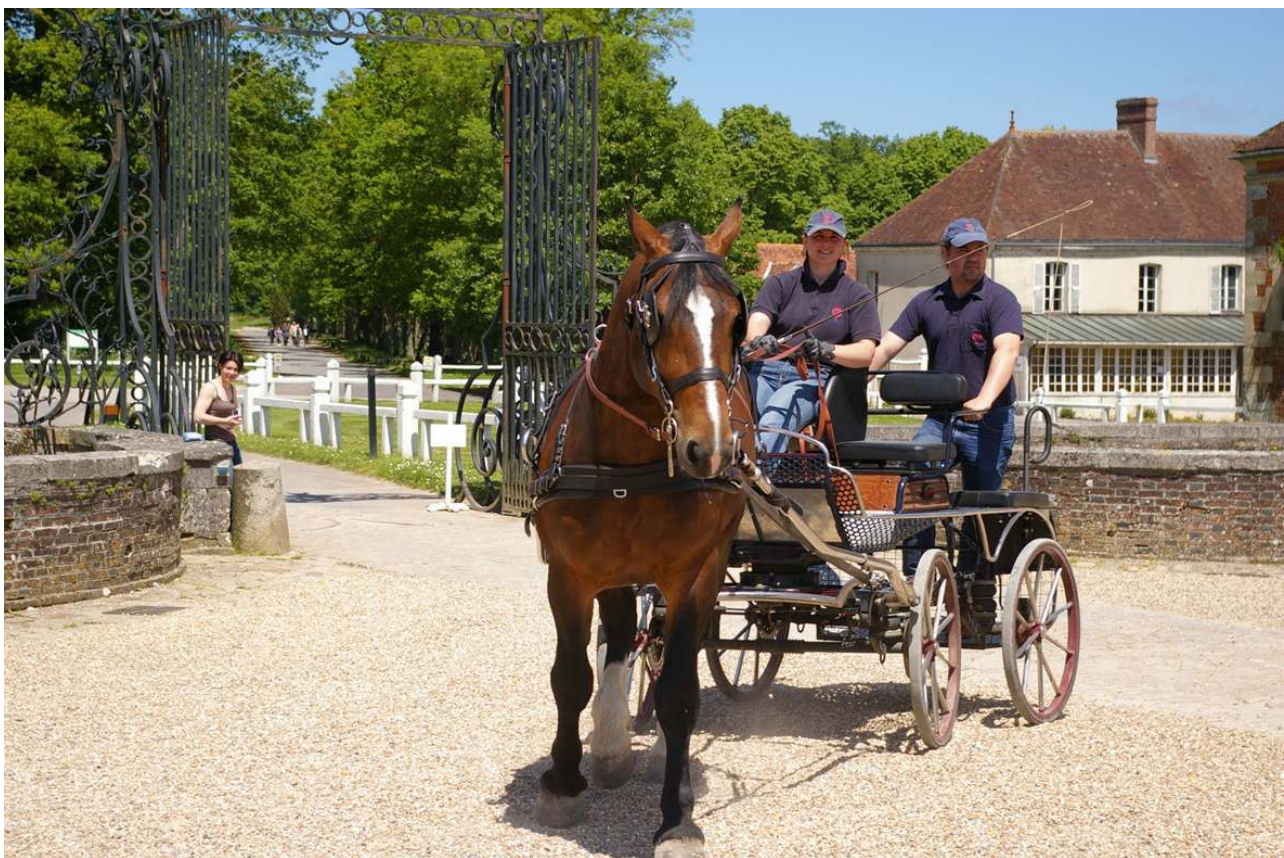

La formation «Attelage » du haras du Pin.

(c) Haras du Pin Tourisme.

\section{Les objets d'art liés à la victoire des étalons sur les champs de courses}

Les trophées équestres gagnés par les premiers pur-sang sont de véritables pièces d'orfèvrerie (théière, cafetière, surtout de table...) réalisées notamment par CharlesNicolas Odiot (fig. 18). 
Figure 18

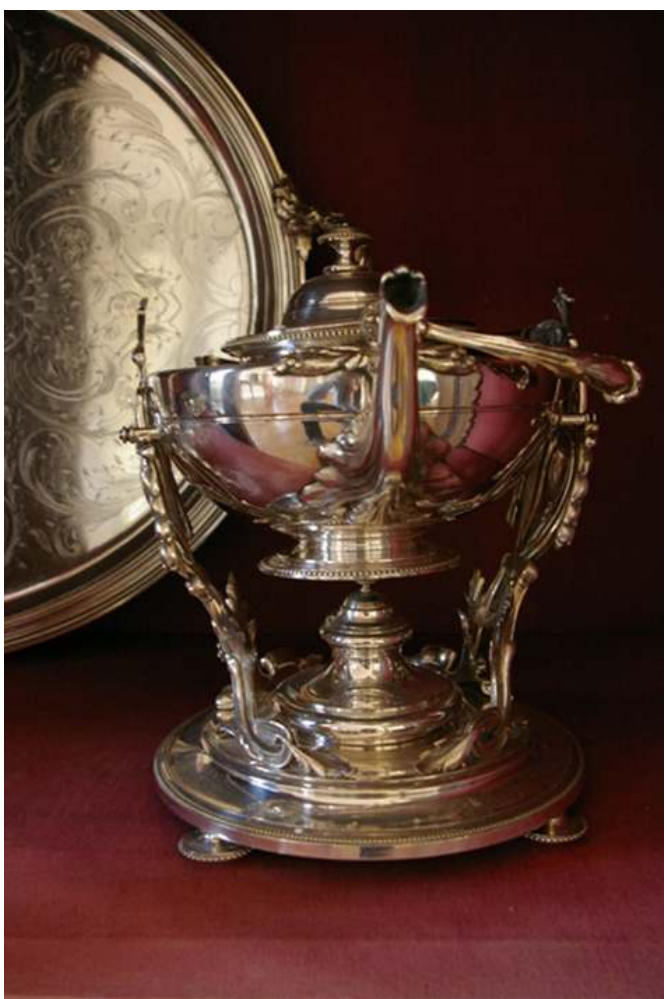

Bouilloire de table, argent et ébène du XIXe siècle, dim. 19 × 30 × 23 cm, poinçon de Charles Christofle. (c) Haras du Pin Tourisme.

\section{Les collections liées à la fonction de représentation du haras et les collections « exogènes »}

Les directeurs ont réuni différents objets dans le château, dont les cadeaux officiels offerts lors de visites de délégations étrangères. On trouve également des gravures anglaises représentant des scènes de chasse à courre ou des départs de courses. Ce sont toutefois les quatre tapisseries du XVII ${ }^{\mathrm{e}}$ siècle dessinées par Simon Vouet qui constituent la pièce majeure du château (fig. 19). 


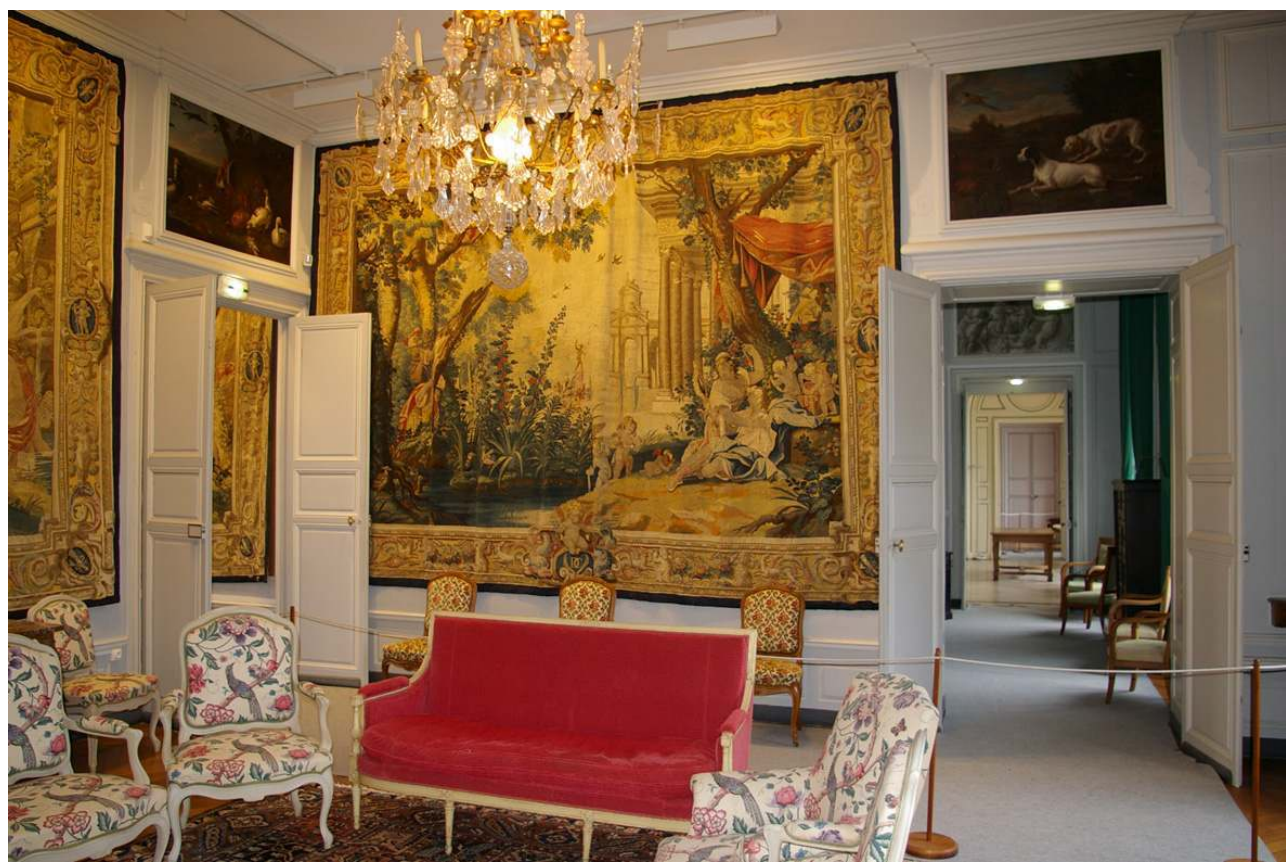

Salon des tapisseries, château du haras du Pin - La Jérusalem délivrée, 4 tapisseries du XVII siècle, cartons de Simon Vouet; lissier : atelier de Raphaël de la Planche, Paris.

(c) Haras du Pin Tourisme.

41 L'écurie $n^{\circ} 1$, l'ancienne écurie des petits étalons réservée aux chevaux de carrosses, est devenue un centre d'interprétation du patrimoine, et l'ancienne école des haras un espace d'accueil, depuis 2006 (fig. 20, fig. 21). Cet espace réunit avant tout des collections propres à l'outil de travail, comme les podiums de sellerie et de maréchalerie, mais aussi des collections exogènes liées au cheval en général, avec les ouvrages anciens mis à disposition par l'Institut français du Cheval et de l'Équitation, ou encore les dons comme la veste de dressage de Dominique Desmées. Le centre a été labellisé « Musée de France » en 2011, et l'association a mis en place une politique d'expositions temporaires comme celle, en 2012, sur le peintre Alfred de Dreux, faisant appel à des prêts d'autres collections. 
Figure 20

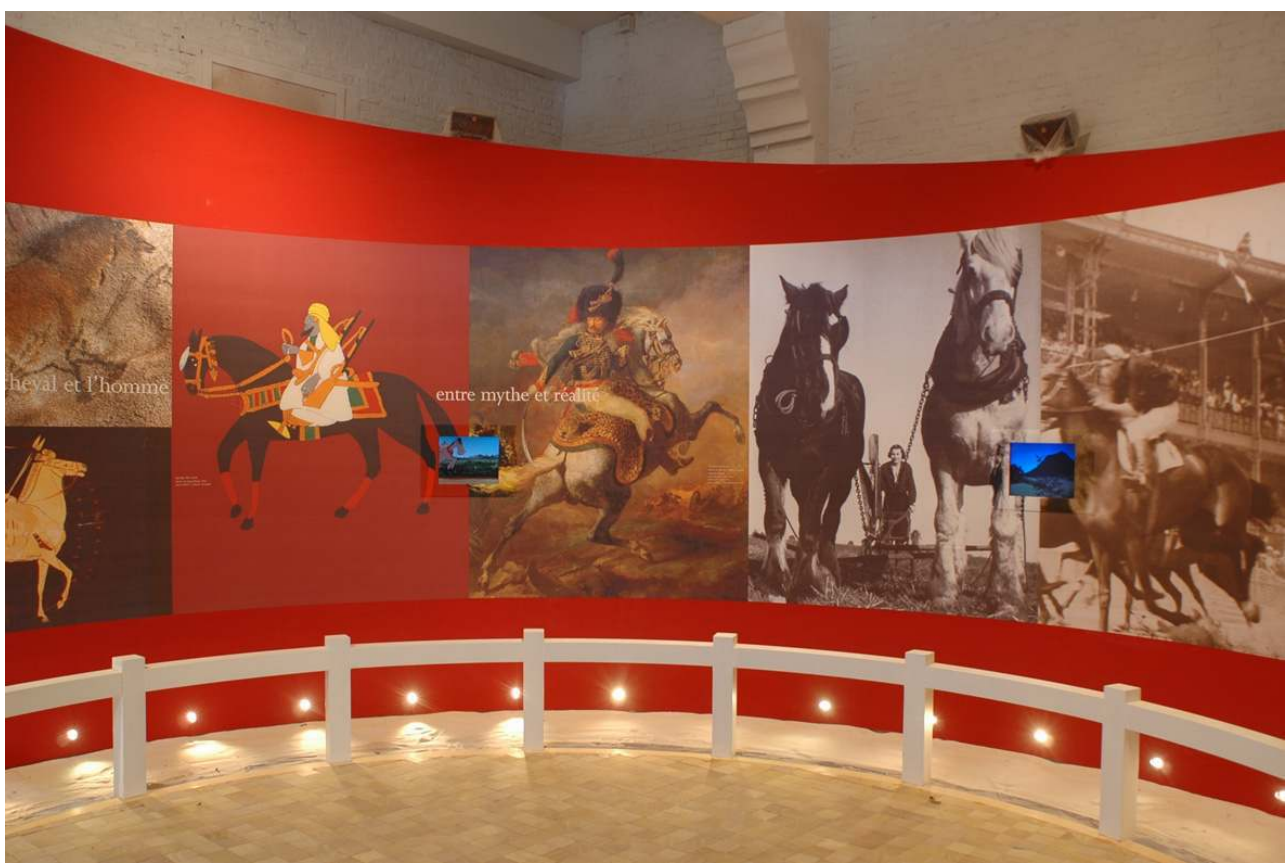

Centre d'interprétation du patrimoine, dans l'Écurie $n^{\circ} 1$ du haras du Pin.

(c) Haras du Pin Tourisme.

Figure 21

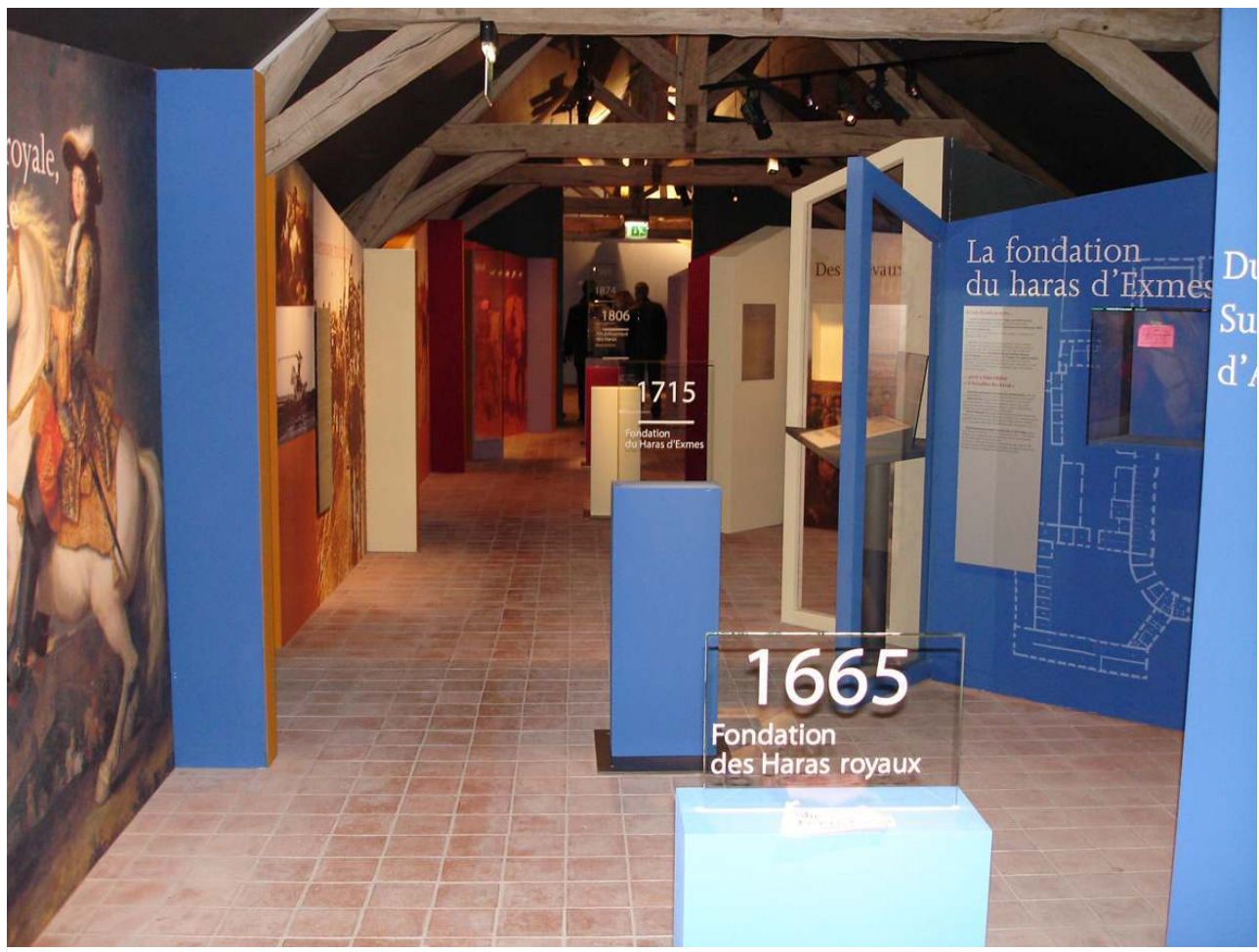

Centre d'interprétation du patrimoine, espace Histoire dans l'Écurie $n^{\circ} 1$ du haras du Pin.

(c) Haras du Pin Tourisme. 


\section{Les enjeux de la conservation des collections patrimoniales du haras du Pin}

42 Aujourd'hui, l'inventaire inachevé des collections patrimoniales du haras du Pin se caractérise par son hétérogénéité. Une sélection des objets sera nécessaire avant de rédiger cet inventaire, de l'informatiser, pour un véritable catalogage des données et un accès rapide à celles-ci. Cet inventaire nous permettrait également de connaître le volume précis des collections. Informatisé, ce serait un outil de contrôle des entrées et sorties d'objets et œuvres d'art du haras, offrant aussi la possibilité de répondre à des demandes de prêt, de faciliter le travail des chercheurs et de susciter une mission de valorisation culturelle à laquelle doit répondre le haras (édition, exposition...).

43 La difficulté de la gestion et de l'étude des collections patrimoniales du haras du Pin est liée à différents facteurs :

44 - l'inventaire et la conservation d'objets, avec la multiplicité des matériaux, relèvent de connaissances scientifiques et techniques très larges,

45 - la diversité des acteurs sur le site même, l'absence d'un statut juridique privilégiant une gestion uniforme sur le site,

46 - l'absence d'un responsable des collections pour coordonner tout ce travail de gestion et d'étude,

47 - la difficulté de concilier la dimension professionnelle du haras avec la dimension patrimoniale: qu'est-ce qui est utilisé ? Qu'est-ce qui ne l'est plus? Ceci doit-il être conservé et valorisé ?

48 - la question de la place de la dimension patrimoniale dans la formation des métiers?

\section{Les enjeux de la valorisation des collections au haras du Pin}

49 Par rapport au « Projet du Pin » qui doit voir émerger une structure collégiale entre l'État et les collectivités territoriales, la valorisation est à la fois culturelle et économique. Dans un domaine préservé mais menacé aujourd'hui par une décharge de déchets industriels à $8 \mathrm{~km}$ de là, à Nonant-le-Pin, le haras du Pin peut être un élément de développement durable sur son territoire en protégeant son environnement, en lui redonnant les leviers économiques pour qu'il puisse vivre en accord avec sa communauté, tant avec les personnes qui travaillent sur le site qu'avec la population locale.

50 Sur le plan touristique, après avoir mis sur pied le centre d'interprétation du patrimoine et l'espace d'accueil, le haras du Pin, et surtout le domaine du Pin, a besoin d'accueillir des visiteurs en mettant à leur disposition des possibilités d'hébergement, en leur proposant des balades à cheval (avec différents niveaux d'équitation) et une offre de loisirs diversifiée. Il s'agit d'innover et d'agir à partir de la ressource que représente le haras pour son territoire, en conciliant économie et patrimoine à partir d'un secteur d'activité majeur, le cheval (pôle de compétitivité en Normandie), et de secteurs qui lui sont liés, la formation, le tourisme et la culture. Pour cela, il faut accepter, comme l'ont écrit Jean-Pierre Babelon et André Chastel dans La notion de patrimoine que «ce 
patrimoine se reconna[isse] au fait que sa perte constitue un sacrifice et que sa conservation suppose des sacrifices ${ }^{2} »$.

Par rapport au thème «le cheval et ses patrimoines", objet d'étude depuis 2011 de la direction générale des Patrimoines, le haras du Pin constitue donc une unité patrimoniale culturelle unique et territorialement forte.

Ainsi, peut-on dissocier au haras du Pin le patrimoine écrit et iconographique du patrimoine équestre proprement dit? Que seraient les collections mobilières, notamment les voitures hippomobiles, sans le cheval ? Il s'agit bien de relier, en tout état de cause, l'immatériel au matériel, et c'est sans doute le patrimoine ethnographique qui en est la plus parfaite illustration; quelle est la valeur d'un objet comme le couteau demi-lune du sellier sans le savoir-faire et la formation du sellier et le contexte du haras ? Jean-Pierre Digard l'a affirmé, à la suite de Leroi-Gourhan : « il est impossible de séparer les objets des outils, des connaissances et des savoir-faire, des gestes.... $"$ ".

$\mathrm{Si}$ on reprend La grammaire des civilisations de Fernand Braudel, le cheval et ses patrimoines au haras du Pin se caractérisent par un territoire situé dans la première région d'élevage du cheval en France, une société propre au haras, une économie et une mentalité collective. Il est donc primordial d'envisager les collections patrimoniales du haras du Pin dans leur contexte d'origine, d'essayer de maintenir l'unicité l'adéquation, l'harmonie? du lieu avec ses collections, de les préserver même si son avenir est incertain et surtout, ne pas «externaliser » les collections des bâtiments et du domaine en leur gardant ce qui fait l'essence même du haras, le cheval, et avant tout, l'étalon.

\section{NOTES}

1. - Voir le site: http://www.culture.gouv.fr/public/mistral/caran_fr? ACTION=CHERCHER\&FIELD_1=REF\&VALUE_1=B-002476.

2. - BABELON, Jean-Pierre, CHASTEL, André. La notion de patrimoine. Paris : Liana Levi, $1994,\left(1^{\mathrm{e}}\right.$ éd. Revue de l'art, 1980), p. 101.

3. - DIGARD, Jean-Pierre. «Les cultures équestres européennes: définitions, tectonique et implications patrimoniales » [document électronique]. In situ. Revue des patrimoines, $\mathrm{n}^{\circ} 18$. Paris : Ministère de la Culture et de la Communication., août 2012, p. 5 : http://insitu.revues.org/9655.

\section{RÉSUMÉS}

Par la volonté de Louis XIV, et depuis le Siècle des Lumières, le haras du Pin représente une empreinte patrimoniale forte sur un territoire marqué par le cheval. Tout ce qui fait aujourd'hui la diversité des collections du haras du Pin a été réuni par l'homme pour élever des chevaux. Le 
cheval est le dénominateur commun des collections patrimoniales du haras du Pin, preuve de l'intérêt animal, paysager, architectural, ethnographique, mobilier - dont le patrimoine hippomobile - et muséographique, de ces collections. Comment se caractérisent ces collections, comment se sont-elles constituées au haras du Pin? On distinguera les collections propres à l'outil de travail, celles liées à la fonction de représentation du haras et enfin, les collections exogènes. En considérant la diversité patrimoniale des collections du haras du Pin, nous essaierons de déterminer quels sont aujourd'hui les enjeux de leur conservation et de leur valorisation.

The cultural and historical variety of the collections at the haras du Pin national stud: the challenges of their conservation and value enhancement. Since the Age of Enlightenment and on the Royal will of Louis XIV, the haras du Pin national stud remains to this day a strong, heritage focal point, in a region of significant importance to the horse. The diversity of the collections at the stud has been brought together by man in order to promote the horse. The horse is the common factor in these collections, the evidence of the interest in the animal is shown through his presence in the landscape, in the architecture, culture and furniture, in the horse-drawn carriages and museum. How are these collections characterised? How are they defined at the national stud? We can identify them clearly - through the workmanship at the stud, by the public relations activities and finally those collections external to the stud. Given the diversity of these heritage collections at the haras du Pin, we will try to determine today the challenges of their conservation and the enhancement of their value.

\section{INDEX}

Mots-clés : haras, cheval, étalon, collections, patrimoine, objets, savoir-faire, voitures hippomobiles, sellerie, maréchalerie, nouveaux métiers, conservation, valorisation

\section{AUTEUR}

\section{MURIEL MENEUX}

responsable de l'Association pour le développement et le rayonnement du haras du Pin muriel.meneux@wanadoo.fr 